\title{
Caracterización del estado ecológico del recurso forestal en la Reserva Natural Llanos de Karawala
}

\section{Characterization of the ecological status of the forest resource in the Llanos de Karawala Nature Reserve}

\author{
Juan Asdrúbal Flores-Pacheco ${ }^{1}$, Elvy Garvin Knight Santiago ${ }^{2}$ \& Antonio José \\ Obregón Hernández ${ }^{3}$
}

(Bluefields Indian \& Caribbean University)

\begin{abstract}
RESUMEN
En este trabajo se evalúa el papel ecosistémico y funcional de los árboles en el sostenimiento y en la conservación de la reserva natural Llanos de Karawala en la cuenca del Río Grande de Matagalpa en la Región Autónoma Costa Caribe Sur (RACCS) de Nicaragua. La caracterización de las especies forestales de extracción selectiva se hizo mediante estudios ecológicos y socioeconómicos. Esta cobertura arbórea tiene gran importancia socioeconómica, porque provee a los productores especies de usos múltiples: leña, alimento para el ganado, madera para construcciones rurales; brinda además al productor otros servicios: provisión de sombra y conservación de suelos. Se logró conocer que las especies de más demanda son las de mayor extracción: pino Caribe (Pinus caribaea), caoba del Atlántico (Swietenia macrophylla) y Santa María (Calophyllum brasiliense), de las cuales dos son protegidas por la Ley de Veda Forestal. Los pobladores de la zona tienen un amplio conocimiento local acerca de la cobertura arbórea presente y la manejan para obtener productos y servicios. Además, la cobertura arbórea sirve como importante recurso alimenticio y refugio para la flora y fauna silvestre. En base a la caracterización de la cobertura arbórea y sus roles ecológicos y socioeconómicos, se discuten alternativas sobre cómo conservar y manejar la cobertura arbórea de manera compatible con los objetivos de producción y conservación. Toda esta información debe ser pasada a decisores comunales y regionales para una intervención oportuna.
\end{abstract}

Palabras claves: ecología forestal, aprovechamiento, reserva natural, comunidad indígena. Nicaragua-Costa Caribe

Recibido: 22 de febrero del 2019

Aceptado: 28 de abril del 2019

\footnotetext{
${ }^{1}$ Doctor Conservación y Uso Sostenible de Sistemas Forestales, Director de Investigación y Posgrado, Bluefields Indian \& Caribbean University, email: asdrubal.flores@do.bicu.edu.ni; ORCID https://orcid.org/0000-0001-6553-7202

${ }^{2}$ Licenciado en Ecología de los Recursos Naturales Graduado en BICU, email: eknight@ cukra.com

${ }^{3}$ Licenciado en Ecología de los Recursos Naturales Graduado en BICU, email: aobregon@ @ukra.com
} 


\section{ABSTRACT}

This paper evaluates the ecosystemic and functional role of trees in sustaining and conserving the Llanos de Karawala Natural Reserve in the Rio Grande de Matagalpa basin in the Autonomous Region of the Southern Caribbean of Nicaragua. characterization of forest species of selective extraction, through ecological and socioeconomic studies. This tree cover has great socioeconomic importance because it provides multiple use species to the producers: firewood, cattle feed, wood for rural constructions; and provides other services to the producer: provision of shade and soil conservation. It was learned that the spices most in demand are the species with the highest extraction are Caribbean Pine (Pinus caribaea), Atlantic Mahogany (Swietenia macrophylla) and Santa María (Calophyllum brasiliense) of which two are protected by the Forest Prohibition Law. The inhabitants of the area have extensive local knowledge about the current tree cover and manage it to obtain products and services. In addition, tree cover serves as an important food resource and refuge for flora and fauna. Based on the characterization of tree cover and their ecological and socioeconomic roles, alternatives are discussed on how to conserve and manage tree cover in a manner that is compatible with production and conservation objectives. All this information must be passed on to community and regional decisionmakers for a timely intervention.

Keywords: forest ecology, use, natural reserve, indigenous community, NicaraguaCaribbean Cost

\section{INTRODUCCIÓN}

En Nicaragua existe un total de 77 áreas protegidas, legalmente establecidas bajo un decreto presidencial emitido por el estado o a través de MARENA, y de las cuales solamente el $15.80 \%$ de éstas cuenta con planes de manejo activo, bajo presencia institucional, presupuesto y personal permanente (MARENA - PANIF, 2001). En la reserva natural Llanos de Karawala existe una extensa masa forestal de pino la cual está siendo deforestada a un ritmo acelerado por los mismos pobladores de la zona. En los últimos años, la actividad de la deforestación ha aumentado su nivel de efectos en nuestro país, lo cual ha generado una serie de problemas en la comunidad de Karawala, por la destrucción del hábitat natural y otros sitios naturales de suma importancia para la comunidad, generando impacto negativo al ambiente, por falta de planificación ecológica, y el aprovechamiento descontrolado de los recursos forestales (Knight y Tenorio 2009).

En años anteriores, la reserva natural Llanos de Karawala tuvo afectaciones de diferentes fenómenos naturales de gran magnitud, que provocaron un gran daño irreversible. Hoy en día la población se ha incrementado y con ello la deforestación del recurso (Pinus caribaea) que es una madera preciosa que todos buscan y cortan sin saber el daño que le causa a la reserva natural. Otro factor que influye mucho, en la pérdida de cobertura y regeneración 
natural del bosque es el fuego, pues en la época de verano son muy frecuentes los incendios forestales, ya sean provocados accidentalmente o por el intenso calor.

La deforestación, causada por los fenómenos naturales y por los mismos comunitarios, ha sido y es un problema muy importante que debemos tratar de solucionar, para que la reserva natural Llanos de Karawala pueda recuperar la cobertura boscosa que tenía hace varios años y así se convierta en un pulmón más de nuestro país. La metodología aplicada para llegar a los resultados del estudio se basa en la aplicación de entrevistas, revisiones bibliográficas, muestreos de campo y observación directa en el sitio de estudio. Esto, para la recolección de datos cualitativos y cuantitativos, que permitieran determinar el estado de la situación actual del bosque de pino, ejecutando así una confiable y justa identificación, descripción y evaluación del recurso.

Esta metodología se ajustó a la realidad de la comunidad. En este sentido tomamos en cuenta la aportación de la población local. Esto para confirmar la situación actual de la reserva natural Llanos de Karawala, las cual está sometida a la presión extractora. Para eso se realizó talleres participativos, entrevistas aplicadas, muestreos de campo, observación directa; además, los documentos investigativos fueron los principales materiales en la elaboración del trabajo y para conseguir los datos presentes y anteriores del sitio, y cuyos resultados constituyeron las primeras fuentes de información de la investigación.

Con el presente estudio se pretende identificar los principales daños causados por el aprovechamiento forestal, para brindar a los comunitarios información sobre el grado de afectación del recurso, y así elaborar propuestas de manejo forestal, en la Reserva natural Llanos de Karawala, que sirva a las autoridades comunales, territoriales y regionales para la toma de decisiones relevantes en cuanto a la protección, manejo y uso sostenible del recurso.

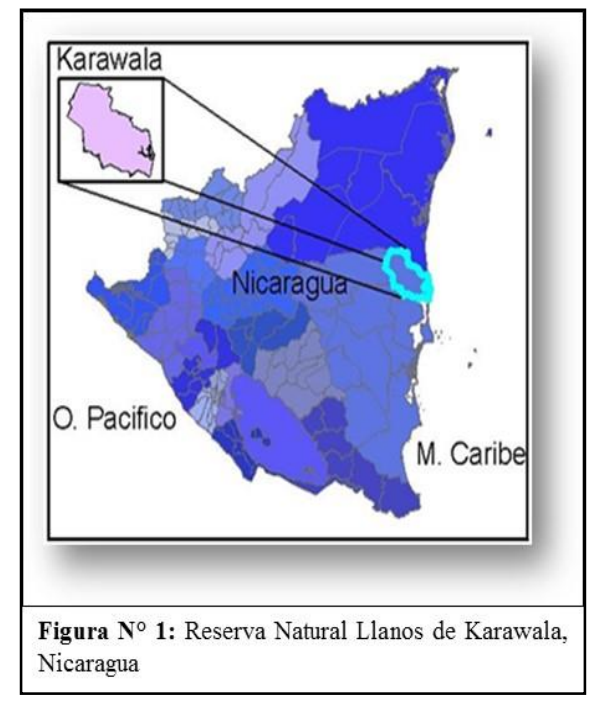




\section{DISEÑO METODOLÓGICO}

\section{Ubicación y descripción del sitio de estudio}

El área de estudio de esta investigación es de $74.4 \mathrm{~km}^{2}$ aproximadamente, territorio hereditario de la comunidad de Karawala (Knight, 2001). Esta comunidad indígena está asentada en la orilla de un pequeño caño llamado Walangwas que tiene afluencia con el río Grande de Matagalpa. Según su posición geográfica se encuentra localizada en las altitudes N $12^{\circ} 55^{\prime} 89.6^{\prime \prime}$ y latitudes W 83 34' 62.6". Se sitúa a $134 \mathrm{~km}$ al norte de Bluefields, y a 7 $\mathrm{km}$ al oeste de la barra del río Grande de Matagalpa, propiamente en el costado este del caño Walangwas. Limita al norte con la comunidad de Sandy Bay Sirpi, al sur con la comunidad de Kara, al este con la comunidad Barra de Río Grande, y al oeste con el caño Walangwas, en el municipio de la Desembocadura de Río Grande, Región Autónoma Costa Caribe Sur (RACCS) en Nicaragua. El área de estudio está ubicada en bosque conífero, aproximadamente a cinco kilómetros norte de la comunidad de Karawala, y a 2.5 kilómetros de la microcuenca de Karawala. El área seleccionada para el estudio es propiedad de la comunidad indígena ulwa de Karawala. El llano de pino se encuentra asociado con palmas muy cercas de extensos bosques latifoliados, rodeados a su vez de bosques de galería y manglares (MARENA /CBA, 2003).

La zona de estudio se caracteriza por presentar un clima tropical húmedo, con dos estaciones (invierno y verano). La precipitación pluvial va de 3,200 a 4,000 mm anuales y la temperatura media es de $26^{\circ} \mathrm{C}$ (MARENA /CBA, 2003).

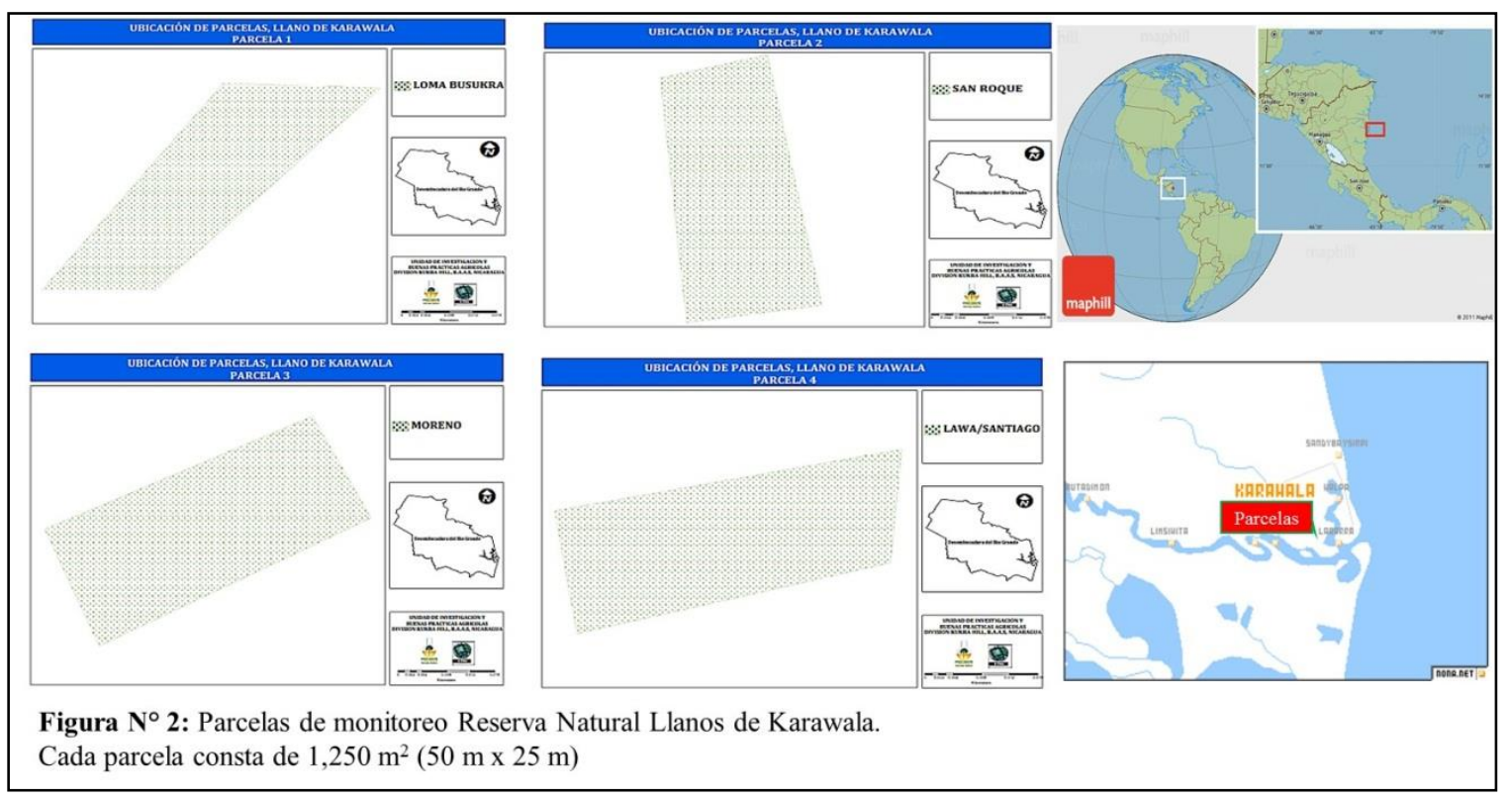




\section{Tipo de investigación}

Este estudio es de tipo descriptivo con corte transversal. Es descriptivo porque se señala cada una de las potencialidades de la masa boscosa y los daños a los que se ha visto sometida por la actividad antropogénica. Es también de corte transversal, porque la recolección de datos sobre el llano de pino se llevó a cabo en un tiempo dado.

\section{Enfoque de la investigación}

Este estudio se basa en un enfoque mixto, ya que, con respecto a las posiciones, ubicación de las muestras de campo y las mediciones de los árboles, entre otras características descriptivas, generaron datos numéricos, los cuales fueron operacionalizados con estudios cuantitativos, aunque también se hicieron descripciones y evaluaciones de las condiciones en que se encuentran los árboles de pino, a través de la observación directa, lo cual corresponde al enfoque cualitativo. Los enfoques cualitativos y cuantitativos se combinaron en el proceso de la investigación, o al menos en la mayoría de sus etapas.

Durante la primera visita al campo se desarrolló un "Taller de Diagnóstico Participativo" de dos días, con un grupo de 25 personas integrado por: líderes comunales, aserradores locales, dueños de fincas y ancianos considerados excelentes conocedores de la zona. Durante el taller se aplicó una entrevista, con el fin de valorar sus conocimientos previos sobre el uso adecuado del sitio, principalmente en torno a la población de pino existente en diferentes zonas del llano, el uso racional y la protección del área; también para conocer mejor los principales daños en la masa boscosa, por causas naturales y antropogénica. Luego, toda la información adquirida fue consolidada de manera conjunta, considerando que los participantes, al tener un conocimiento apropiada sobre el tema de la investigación, podrían aprovechar el recurso de una manera racional en el futuro.

Estas personas fueron seleccionadas según su cargo o función que desempeñan en la comunidad, puesto que así logramos obtener información acerca de lo que ellos piensan y consideran en torno a cómo están utilizando el recurso en la Reserva. Ya sea por los factores naturales que afectaron dicha reserva o por el daño causado por los mismos pobladores que, sin tener ningún conocimiento -o aun teniéndolo- aprovechan de manera inadecuada los recursos forestales, acentuando así la degradación de los suelos, la disminución de la masa boscosa y, por ende, de los ríos a causa de la tala de los árboles en sus orillas. 
El universo de este estudio es el espacio geográfico de la comunidad de Karawala. Para la selección de la muestra se utilizó el método, no probabilístico, que se encuentra en el libro de metodología de investigación (Sampiere: 2003) ya que fueron hechas 25 entrevistas de manera individual, a igual número de personas, con el fin de conocer su nivel de conocimiento sobre la situación actual a la que se está enfrentando la población de pino en esta área. Los participantes en estas entrevistas fueron seleccionados en calidad excelentes conocedores de la zona de: líderes comunales, aserradores locales, dueños de fincas.

\section{Identificación de los bienes y servicios}

En el segundo viaje se desarrolló la identificación de los bienes y servicios existentes en la comunidad, a través de entrevistas a los líderes comunales, consejos de ancianos y algunos pobladores que realizan actividades diarias en el llano: aserradores locales y los dueños de fincas.

\section{Diagnóstico participativo de la condición del sitio}

\section{Aplicación de entrevistas}

Esto se realizó para considerar la participación de los comunitarios, sus conocimientos y puntos de vistas sobre la pérdida de la masa boscosa del bosque de pino y las principales causas que la provocaron.

\section{Aplicación de la metodología FODA}

Es un método para la planificación estratégica basado en el análisis sistemático de los factores internos y externos que afectan el desempeño de un recurso potencial; la cual fue aplicada con el fin de conocer las diversas causas que determinan; cuál de las actividades que provocan mayor afectación en llano de pino y así poder determinarlos condiciones que se encuentran la población de pino para poder desarrollarlos. Fue aplicado con el fin de conocer el origen de las actividades que provocan mayor afectación en el llano de pino. Esto, para determinar las condiciones en que éste se encuentra y así elaborar estrategias al respecto. Para el desarrollo de esta metodología se trabajó con grupos focales integrados por 25 personas que cumplían con las siguientes características: líderes comunales, aserradores locales, dueños de fincas, excelentes conocedores de la zona. 


\section{Aplicación de muestras en el campo}

El llano de pino en la comunidad de Karawala tiene un área total de 2,000 hectáreas de bosques de pino, situados en distintas zonas del llano. Allí se hicieron cuatro muestras de campo en diferentes zonas del sitio en que se encontraba la mayor población de pino, tomando en cuenta las ubicaciones de los puntos de muestreo que fueron marcados con geoposicionador satelital (GPS). En el sitio en el cual se realizaron las mediciones de la parcela, que consistía en $20 \mathrm{~m}$ de ancho por $50 \mathrm{~m}$ de largo, se empleó el método de barrida haciendo uso de una bitácora de campo que contenía los siguientes datos: especie, diámetro a la altura del Pecho (DAP), altura comercial, altura total.

\section{Cálculo de intensidad de muestreo}

Para calcular la intensidad de muestreo para el área de estudio se utilizó la fórmula propuesta por POSAF/MARENA del 2005, la cual se describe de la siguiente manera: \% IM=TPx N'P/ATR (Ha) x100\% (Fórmula 1). Y en donde:

$\mathrm{IM}=\quad$ Intensidad de muestreo

$\mathrm{TP}=$ Tamaño de la parcela.

$\mathrm{N}^{\circ} \mathrm{P}=$ Número de parcelas.

$\mathrm{ATR}=$ Área total real.

$100 \%=$ Constante porcentual de la fórmula.

$\mathrm{TP}=800 \mathrm{~m}^{2} / 8,000 \mathrm{~m}^{2}=0.1 \mathrm{TP}$

$\% \mathrm{IM}=0.1 \times 10 / 9 \times 100 \%=11 \% \mathrm{IM}$.

Partiendo -para la reserva en estudio- de un área total de 2,000 hectáreas cuadradas (MARENA, 2003), y tomando en cuenta el Inventario Nacional Forestal (INAFOR, 2009), estimamos en $30 \%$ la cobertura forestal de pino. El área efectiva de muestro fue calculada en 66 hectáreas cuadradas. Los puntos fueron seleccionados por Muestreo Aleatorio Simple, en función de la accesibilidad y la extracción de madera en el sitio. 


\section{Obtención de altura total, altura comercial y diámetro a la altura del pecho (DAP)}

Para la obtención de datos de altura total y altura comercial se utilizó un hipsómetro y un clinómetro, para medir cada árbol encontrado en la parcela de muestreo. El diámetro del árbol se mide a 1.30 metros sobre el nivel del suelo, lo cual se conoce como diámetro a la altura del pecho (DAP). Para la medición del diámetro de los árboles a la altura del pecho (DAP) se utilizó una cinta métrica y diamétrica.

\section{Regeneración Natural}

En cada parcela de muestreo se estableció al azar una subparcela de regeneración, con dimensiones de diez metros de ancho y 15 metros de largo, en la cual fueron medidos árboles de pino menores de diez centímetros de DAP, para los brizales, y para los latizales fueron medidas las plantas mayores de 0.5 - 1 metro de altura.

Cuadro 1. Operacionalización de las variables a evaluar

\begin{tabular}{|c|c|c|c|}
\hline Variable & Método & Frecuencia & Unidades \\
\hline \multicolumn{4}{|c|}{ Fase de entrevistas y encuestas } \\
\hline \multirow[t]{2}{*}{$\begin{array}{l}\text { Colecta de información } \\
\text { de campo con los } \\
\text { comunitarios }\end{array}$} & Entrevistas & $\begin{array}{l}\text { Una por } \\
\text { cada } \\
\text { miembro del } \\
\text { consejo de } \\
\text { ancianos }\end{array}$ & Adimensional \\
\hline & Encuestas & $\begin{array}{l}\text { Pobladores } \\
\text { voluntarios }\end{array}$ & Adimensional \\
\hline \multicolumn{4}{|c|}{ Fase de Campo - Parcelas de monitoreo } \\
\hline Especie & $\begin{array}{l}\text { Identificación con Guía de Especies } \\
\text { Forestales de Interés Económico en } \\
\text { Nicaragua (Herrera, 1995) }\end{array}$ & Única & Adimensional \\
\hline $\begin{array}{l}\text { Diámetro altura de } \\
\text { pecho (DAP) }\end{array}$ & Uso de cinta diamétrica & Única & $\begin{array}{l}\text { Centímetros } \\
(\mathrm{cm})\end{array}$ \\
\hline
\end{tabular}




\begin{tabular}{|c|c|c|c|}
\hline Variable & Método & Frecuencia & Unidades \\
\hline Altura Comercial y Total & $\begin{array}{l}\text { Altura comercial: } \\
\text { Distancia vertical entre el nivel del tocón } \\
(0.25-0.30 \mathrm{~cm}) \text { y la posición terminal de } \\
\text { la última porción comercialmente } \\
\text { utilizable del árbol. } \\
\text { Altura total: } \\
\text { Distancia vertical entre el nivel del suelo y } \\
\text { la yema terminal más alta del árbol. }\end{array}$ & Única & Metros (m) \\
\hline Regeneración natural & $\begin{array}{l}\text { En Parcelas de } 150 \mathrm{~m}^{2} \text { se midió árboles de } \\
\text { pino menores de diez centímetros de DAP; } \\
\text { para los brinzales y los latizales fueron } \\
\text { medidos en plantas mayores de } 0.5-1 \mathrm{~m} \\
\text { de altura. }\end{array}$ & Única & Adimensional \\
\hline $\begin{array}{l}\text { Volúmenes de } \\
\text { extracción de madera }\end{array}$ & $\begin{array}{l}\text { Newton : } \mathrm{V}=\pi / 4 \times \frac{(\mathrm{D} \text { mavor }+\mathrm{D} \text { med }+\mathrm{D} \text { Menor })^{2}}{4} \times \mathrm{L} \\
\text { En base a la fórmula de Newton: }\end{array}$ & $\bigoplus^{0.6 \mathrm{~m}} \begin{array}{l}\text { Única } \\
0.54 \mathrm{~m}\end{array}$ & $\begin{array}{l}\text { metros } \\
\text { cúbicos }\left(\mathrm{m}^{3}\right)\end{array}$ \\
\hline
\end{tabular}




\section{RESULTADOS Y DISCUSIÓN}

Inventario florístico de las principales especies forestales sujetas a extracción selectiva en la Reserva natural Llano de Karawala

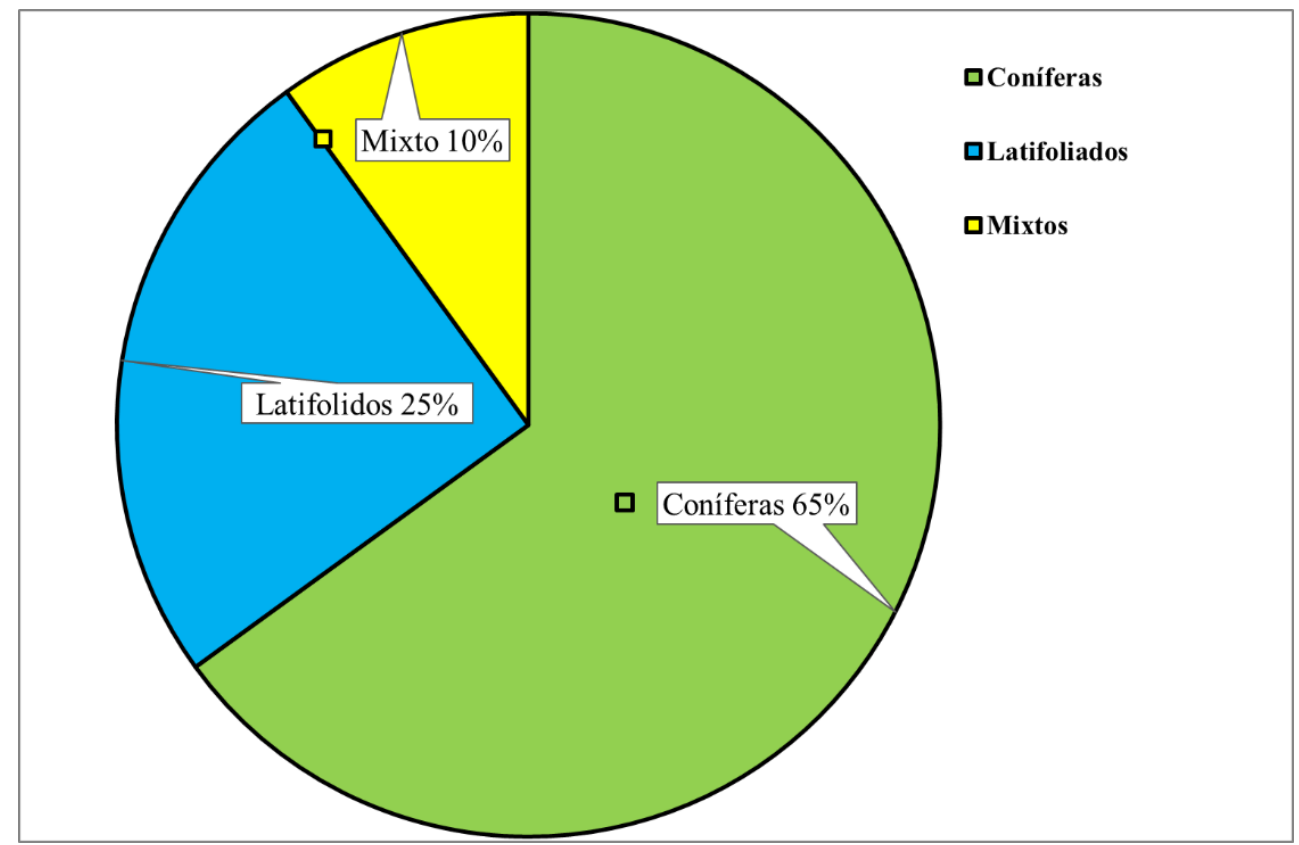

Figura 3. Tipos de bosques y su extensión por tipo de masa forestal en la reserva natural Llanos de Karawala.

La Reserva está localizada al costado norte de la comunidad de Karawala, en las coordenadas geográficas N $12^{\circ} 55^{\prime} 49.3$ " de latitud norte y W $083^{\circ} 34^{\prime} 52.5$ " de longitud oeste. Presenta un relieve extensamente plano con pequeñas variaciones ondulatorias en ciertos puntos, que hace oscilar su altura entre cinco y quince msnm. Constituye la única reserva natural del municipio de la Desembocadura de Río Grande declarada, en los decretos 42-91 del año 199, como reserva natural. Este tipo de área protegida pertenece a la Categoría IV en la clasificación de UICN, Área de Manejo de Hábitat, correspondiente a las categorías reconocidas por la Ley 217 de 1996 (Ley General del Ambiente y los Recursos Naturales) como: a) Refugios de Vida Silvestre, b) Reserva de Recursos Genéticos, y c) Reservas Naturales. Abarca una superficie de 2000 hectáreas donde descansa la amplia llanura de pinos caribaea (Knight y Tenorio, 2009).

La cobertura arbórea que se encuentra en las fincas es el resultado de una serie de decisiones de los productores para retener, mantener, eliminar o sembrar árboles en sus fincas (Harvey, 2001). De la modalidad de actividades que realiza el productor en su finca resulta un impacto sobre la cobertura arbórea. Por ejemplo, la decisión de controlar malezas (sea por medios químicos o manuales) conlleva a la realización de raleo y selección de los árboles en los potreros, afectando la abundancia y distribución de la cobertura arbórea presente. Otras actividades de manejo que afectan negativamente la cobertura arbórea incluyen el uso de fuego; los cambios de usos de suelos (principalmente de charral a 
cultivos o potreros), y la extracción de madera y leña que resulta en una reducción de la cobertura arbórea y de material genético, ya que generalmente se aprovechan los árboles adultos. El uso de químicos para controlar maleza y plagas afecta las especies de regeneración natural y perturba directamente las poblaciones de insectos polinizadores y la cadena alimenticia.

El manejo de la cobertura arbórea en la reserva está influenciado por muchos factores socioeconómicos, como la disponibilidad de empleo y dinero para realizar actividades de manejo o, mayormente, de aprovechamiento de los árboles para abastecer las necesidades económicas de la familia. Por ejemplo, si los pobladores necesitan más ingresos, cambian áreas de bosques a cultivos y/o a pastos para agricultura extensiva. Las fluctuaciones de los precios de la madera también cambian por la especie explotada, ya que al haber un exceso de madera ésta se cotiza barata y en casos extremos se pierde en el campo a esperas de mejores precios; sin embargo, en el otro extremo se vende muy barato, lo cual aumenta el volumen comprado, lo cual genera mayor presión en el recurso y al ecosistema en general.

Factores que influyen en la cobertura arbórea son los diferentes tipos de productores y sus estrategias de aprovechamiento de los recursos. El uso del suelo forestal para agricultura extensiva es diferente de acuerdo con el pensamiento del productor, dependiendo del tamaño de la parcela que aprovecha y el nivel de capitalización, siendo las de mayor consumo las dedicados eminentemente a la extracción de madera para la venta. Además, la cobertura arbórea es diferente entre los distintos tipos de productores agrícolas de subsistencia y madereros, debido al área de explotación y la edad de posesión que tienen sobre las parcelas; es decir, los grupos de pobladores con más años de posesión de la tierra presentaron mayores áreas en conservación y agricultura de rotación que los productores con menos tiempo en la zona (Holdridge, 1978). 


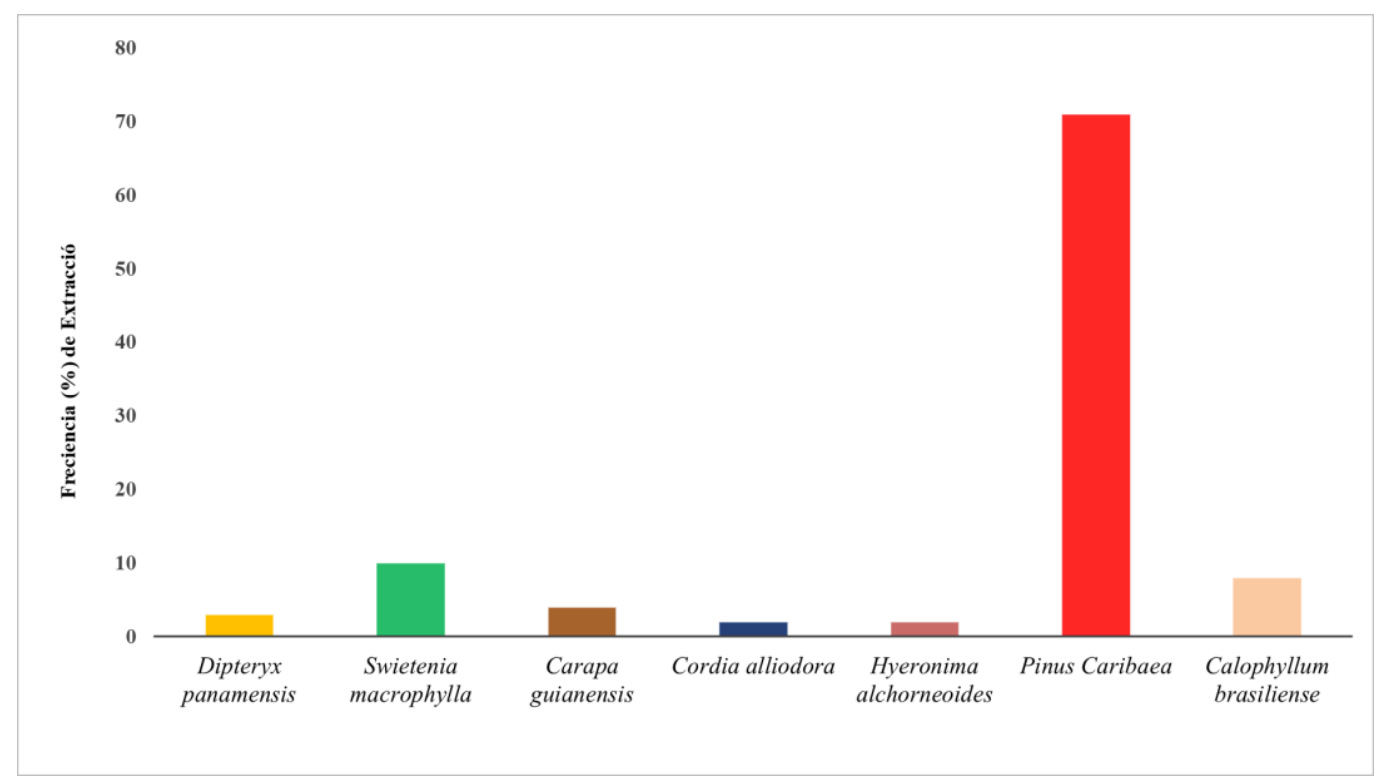

Figura 4. Especies forestales con mayor frecuencia en la Reserva Natural Llano de Karawala.

El paisaje se encuentra altamente intervenido y degradado, producto de la actividad ganadera y agrícola que ha creado un mosaico de pequeños parches aislados, de bosque secundario y bosques riparios, inmersos en una matriz de potreros. Dentro de esta matriz, también hay charrales y árboles dispersos en los potreros. Según el análisis de la foto aérea, la cobertura arbórea está dominada por pasturas que cubren el 56.7\% del paisaje, seguido por bosques secundarios $-15.6 \%$ - y charrales -13.9\%- (INAFOR, 2008).

El cambio del uso de la tierra de vocación forestal a otros usos como la ganadería y agricultura extensiva está incrementado en 1, 583,992 ha; el cambio del uso de la tierra en cultivos temporales y la adición de la deforestación del bosque para su transformación en en 1,907,643 ha de tacotales, ha afectado regiones autónomas, tanto la del Atlántico Norte como la del Atlántico Sur, Jinotega, Matagalpa y Río San Juan. Además, según datos del mapa forestal del 2000 elaborado por MAGFOR, existía en el país un total de 5, 737,198 ha de bosque (se incluye 1, 885,747 ha de áreas protegidas), de acuerdo con el INF 2007-2008, en la actualidad, el inventario reporta que existen unas 3, 254,145 de ha de bosques, aunque sumando las áreas de bosque con las áreas fuera de bosque con árboles, la cifra asciende a unas 7, 572,489 de ha.

Los factores históricos relacionados con el cambio del uso del suelo de las tierras de vocación forestal, relacionados con la deforestación y la degradación del bosque, parecen confirmarse con el Inventario Nacional Forestal, ya que el 54\% de la población entrevistada presenta algún tipo de movilización y solamente un $46 \%$ presenta estabilidad. Por otra parte, la historia de las comunidades rurales indica que han estado sujetas a situaciones o condiciones que afectan su estabilidad y desarrollo, tales como guerra, violencia, desastre natural, conflicto de tenencia de la tierra, entre otros. 
En la actualidad, los procesos de deforestación y degradación de los bosques son temas importantes debido a su contribución a los cambios climáticos. Por esta razón, estos inventarios son claves, porque muestran el aporte que cada sector proporciona en el país en términos de emisiones de gases de efecto invernadero (GEI). Por tanto, los datos nacionales deben reagruparse en categorías estándares o mundiales para que sean comparables con los de otros países. Además, los análisis de biomasa y carbono son de vital importancia para que Nicaragua cuente con información técnica para evaluar su balance anual de emisiones y remociones de gases de efecto invernadero (GEI), debido a los cambios de usos de la tierra.

\section{Estimación volumétrica $\left(m^{3}\right)$ de la extracción de madera de las especies forestales en la reserva natural Llano de Karawala}

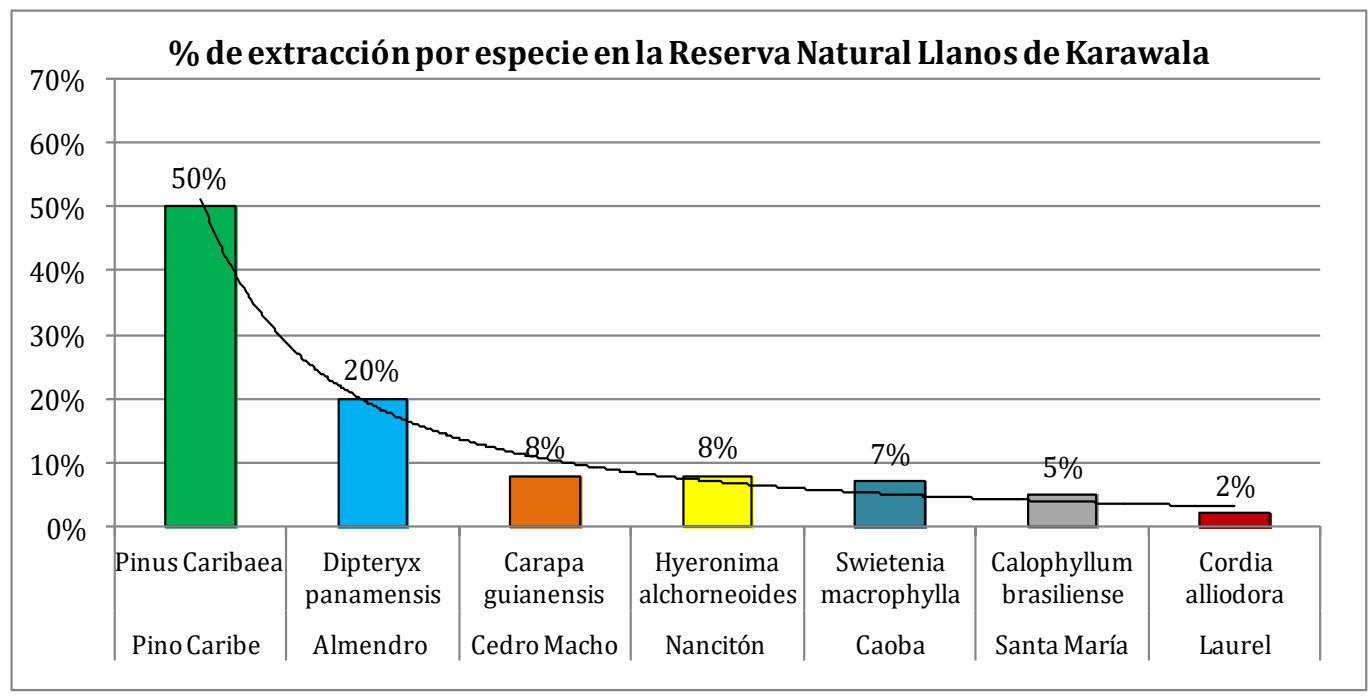

Figura 5. Distribución por clase diamétrica de especies sujetas al aprovechamiento.

Las clases diamétricas son la principal variable del bosque en términos de su uso, aprovechamiento, manejo y otros servicios que brinda el bosque. La legislación nacional relativa al aprovechamiento forestal comercial establece de manera general un diámetro mínimo de corte de $40 \mathrm{~cm}$. (Inventario Nacional Forestal (2007-2008), el establecimiento de mediciones a partir de diez cm de DAP, con el propósito de hacer estudios del desarrollo diamétrico de los árboles, que permita establecer los distintos usos que se podrían obtener del bosque. 
Este gráfico muestra el porcentaje de extracción de las especies en la reserva natural Llanos de Karawala donde la especie que más se extrae es el pino (Pinus caribaea) con un porcentaje (\%) de extracción de 70\% en relación con el área total de la Reserva. Aunque las otras especies, como caoba, almendro, laurel, tengan mayor demanda a nivel municipal, éstas no han alcanzado su diámetro establecido para poder ser aprovechadas; de tal manera, como muestra el gráfico, que el pino es la especie de más población en la zona y con el diámetro establecido de aprovechamiento.

Del 25\% de bosque latifoliados en la reserva natural llano de Karawala encontrados por debajo de los $50 \mathrm{~cm}$ de DAP, existe una distribución homogénea del número de árboles con este DAP, lo que hace indicar que los niveles de intervención son similares. Por otra parte, la cantidad de árboles con DAP arriba de $50 \mathrm{~cm}$ es decreciente. Encontrando menos del $10 \%$ que se ubica entre 20 a $39.9 \mathrm{~cm}$ de DAP, lo que indica que estos árboles se encuentran próximos para obtener un grado de desarrollo óptimo en su crecimiento. Además, existe un $10 \%$ de bosque latifoliados en desarrollo que amerita la aplicación de tratamientos silviculturales para favorecer su crecimiento y sanidad. En los muestreos aplicados, no se midieron diámetros inferiores a los $10 \mathrm{~cm}$.

El 65\% de los árboles del bosque de coníferas se encuentra con un DAP superior a los treinta centímetros, lo cual indica un grado de crecimiento adecuado; el $14 \%$ se encuentra entre 20 y $29.9 \mathrm{~cm}$ de DAP, próximo a alcanzar su madurez. El bosque mixto se caracteriza por poseer árboles latifoliados y de coníferas, los cuales se encuentran, en un 5\% de los árboles, entre el rango de 20 a 29.9 de DAP; este tipo bosque no se ha aprovechado mediante planes de manejo. Por sus características fisiológicas se encuentra en un estado óptimo de desarrollo, además se observa que el $2 \%$ de los árboles se encuentra entre el rango de 30 a $39.99 \mathrm{~cm}$ de DAP, lo que se considera positivo para el mantenimiento de dicho bosque. 


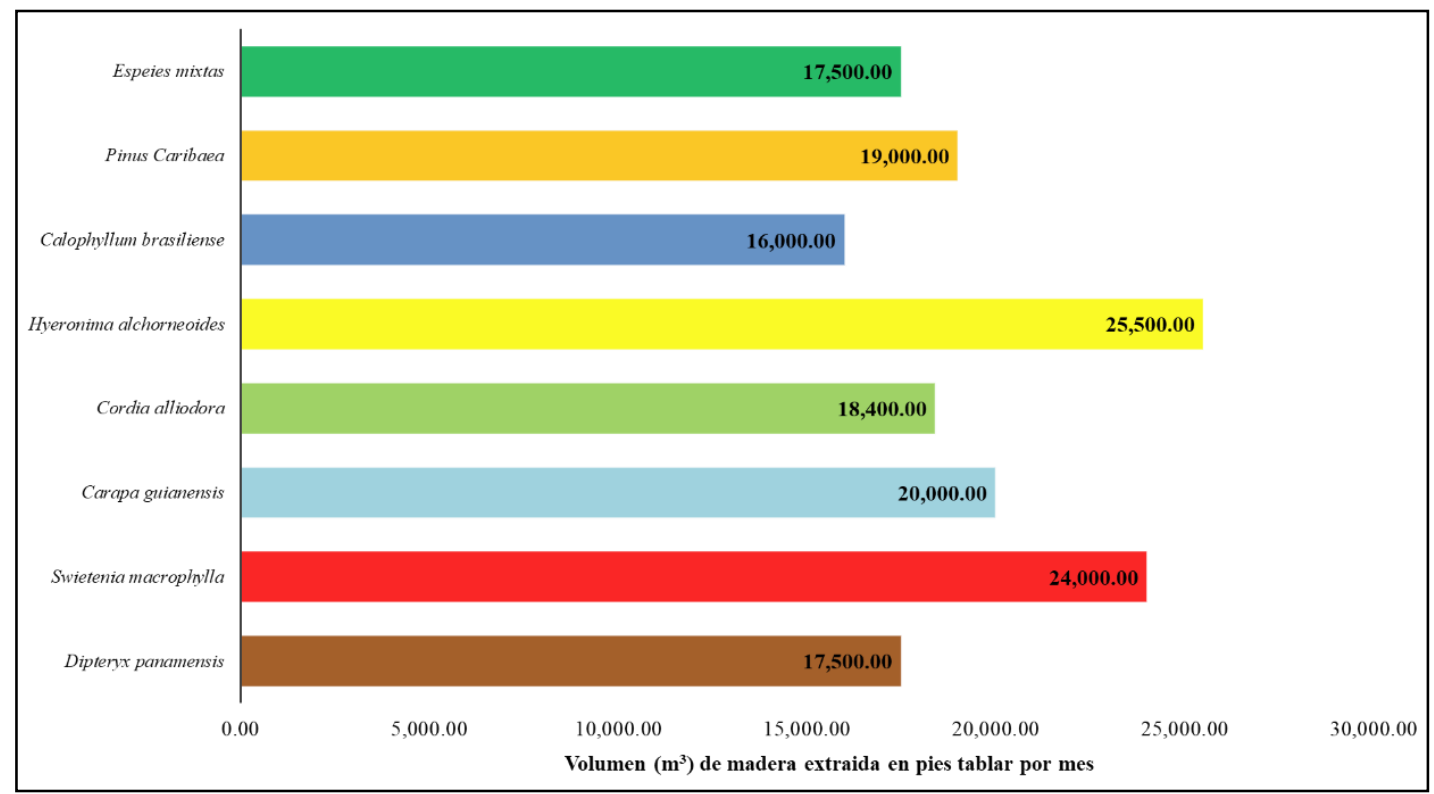

Figura 6. Cantidad de pies tablares extraída por los aserradores locales.

En el momento de la realización de esta investigación se estimó en 25,500 $\mathrm{m}^{3}$ el volumen de extracción de la especie con más aprovechamiento, Nancitón (Hyeronima alchorneoides). Esta es seguida por la Caoba del Atlántico (Swietenia macrophylla) con $24,000 \mathrm{~m}^{3}$; en contraste, la especie conocida como Santa María (Calophyllum brasiliense) es la que presenta menor extracción con $16,000 \mathrm{~m}^{3}$ por año.

En la actualidad se cuentan con pocos bosques de pino no intervenidos en la reserva natural Llanos de Karawala. Los bosques de pino en superficie se mantienen en extensión, pero presentan deterioro en su calidad y densidad. La explotación potencial de los bosques de pino oscila desde $0.5 \mathrm{~m}^{3} / \mathrm{ha} / \mathrm{año}$, en los sitios más pobres, y hasta $10 \mathrm{~m}^{3} / \mathrm{ha} /$ año en los mejores sitios.

Sin embargo, debido a las presiones del crecimiento poblacional, que aumenta la demanda de leña y la extracción de madera tanto legal como ilegal, así como las necesidades de empleos dentro de la comunidad de Karawala, los aserradores locales dependen del bosque y de la madera de pino. Por eso, en la actualidad casi no existen bosques de pino sin intervenir; y aunque se mantiene la superficie, se presenta deterioro en su calidad y densidad, predominando los bosques ralos $(72 \%)$, como producto de la explotación no sustentable, los incendios forestales recurrentes, las plagas y las malas técnicas de extracción de madera (Expresión Personal, Ferlan Santiago). 
Servicios ecológicos proporcionados por las especies forestales identificadas en la reserva natural Llanos de Karawala

\section{Servicios ecológicos}

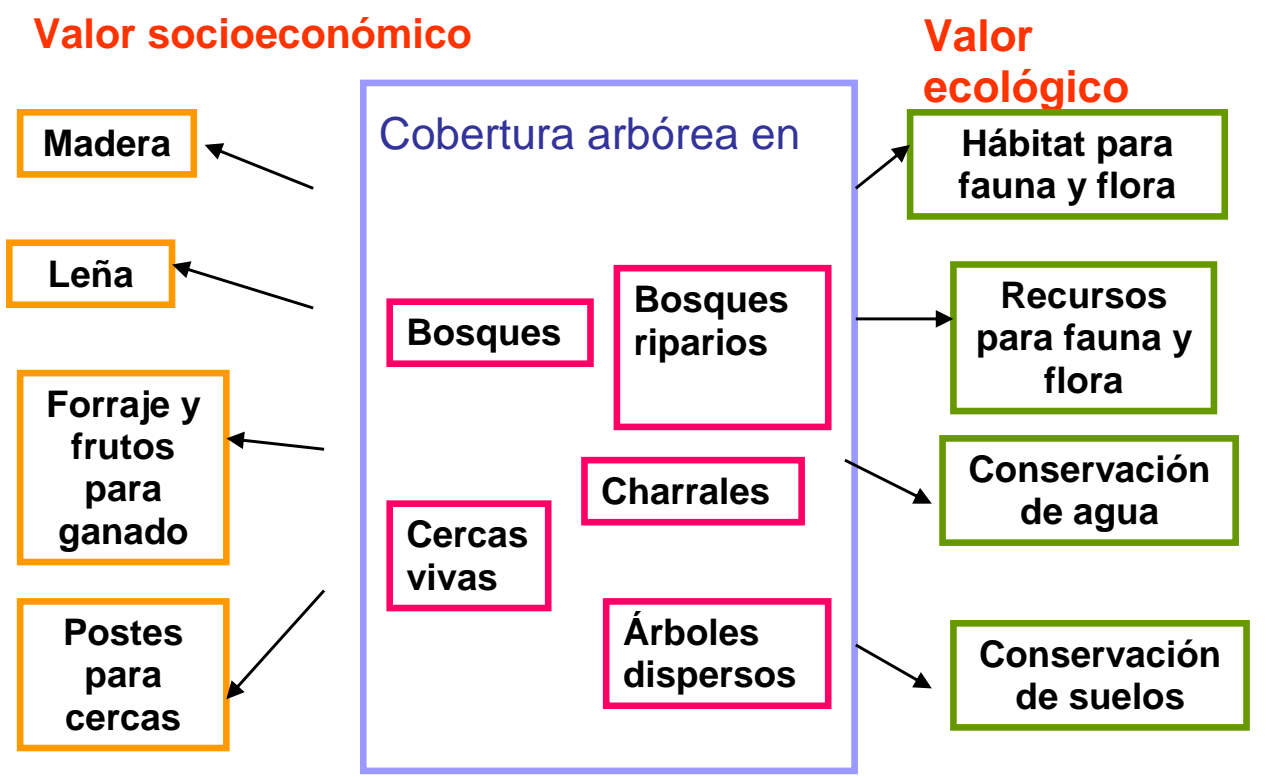

Figura 7. Valor socioeconómico y ecológico de la cobertura arbórea en la reserva natural

Los servicios del bosque de pino para la comunidad Karawala son muy amplios y varían, desde su aprovechamiento para actividades recreativas y turísticas, hasta las funciones vitales para la existencia de la humanidad, como son el mantenimiento de los ciclos hidrológicos y los ecosistemas, hasta la fijación de carbono necesaria para la producción de oxígeno entre otros.

La cobertura arbórea tiene un valor ecológico importante al proveer refugio y nichos para muchos animales cuyos hábitats originales han desaparecido, incluidos algunos de importancia económica como las especies cinegéticas. Además, esta cobertura funciona como un conjunto de corredores que facilitan el movimiento de los animales, también como zona de amortiguamiento y para mejora las condiciones microclimáticas locales. 
La cobertura arbórea en sistemas forestales y silvopastoriles de algunas fincas ubicadas en esta reserva natural tiene también valor ecológico para la fauna. Las cercas vivas son muy escasas, parecen funcionar como corredores biológicos entre parches de bosques para algunas especies de distribución amplia, como las aves y los murciélagos, que las utilizan para trasladarse entre sus áreas de alimentación y de descanso. Igualmente, los potreros con alta cobertura arbórea ayudan a refugiar y alimentar especies importantes para la regeneración natural de estos hábitats (polinizadores y dispersores de semillas). En fin, todos los tipos de cobertura arbórea presentes en el paisaje aportan recursos y mejoraran la conectividad a diferentes escalas, ayudando al mantenimiento de una proporción importante de la biodiversidad original (Casasola, 2000).

Además de su importancia para la conservación de la biodiversidad en los bosques de coníferas, latifoliados y mixtos, los árboles cumplen funciones ecológicas de protección del suelo, disminuyendo los efectos directos del sol, el agua y el viento (Yung, 1989; Montagnini et al., 1992; Fassbender, 1993).

\section{Manejo Forestales}

Con relación al aprovechamiento del bosque en la comunidad de Karawala, los datos obtenidos en las entrevistas y en el taller: un 56\% de los comunitarios muestran una alta conciencia a favor de la conservación y uso racional de los mismos, pero hay un $44 \%$ que todavía no han entendido la importancia que existe en el bosque de pino. La universidad URACCAN impartió un diplomado en las cinco comunidades de la Desembocadura de Río Grande, en el cual se hace énfasis en esta visión del bosque. Un resultado de este diplomado fue la elaboración de un plan de manejo de los extensos bosques de pino con que cuentan estas comunidades y que ahora los comunitarios del municipio de la Desembocadura de Río Grande han resaltado, como un objetivo de este plan la importancia de su implementación.

Entre los criterios que hay que considerar alrededor de la implementación de planes de manejo comunitarios estaría revisar las buenas y malas experiencias de forestaría comunitaria tanto en nuestra región como en nuestros municipios del país. Basándose en esta última experiencia se puede deducir que el fin de la madera extraída con la implementación del plan de manejo debería ser cuidadosamente estudiada, puesto que esto tiene implicancia en la cantidad de recursos que se necesitan para un proyecto de esta naturaleza, considerándose que puede variar, de extracción de madera en rollo, hasta la obtención de producto terminado en forma de muebles, o simplemente de madera aserrada y cepillada. 
La reserva natural Llanos de Karawala tiene las ventajas de estará directamente ligada a los objetivos de conservación de áreas protegidas y sus interconexiones. Asimismo, considerando que existe el criterio de la experiencia previa de los líderes comunitarios en formación de brigadas contraincendios con jóvenes de la misma comunidad, forman que estas actividades sean exitosas y que sirvan para crear conciencia, en las nuevas generaciones, sobre la necesidad de conservar el recurso forestal del llano de Karawala y el bosque de pino. Aunque muchos de estos servicios no cuentan con el reconocimiento de la función que desempeñan para el mantenimiento de la reserva natural (esto debido a la falta de apoyo por parte de las instituciones encargadas de cuidar el estado natural del bosque), sin embargo es necesario orientar y sensibilizar a los líderes comunales, centros educativos, iglesias y a los aserradores locales, para que vaya surgiendo el interés de cuidar, proteger y mejorar las condiciones del estado natural del bosque de pino en el llano de Karawala.

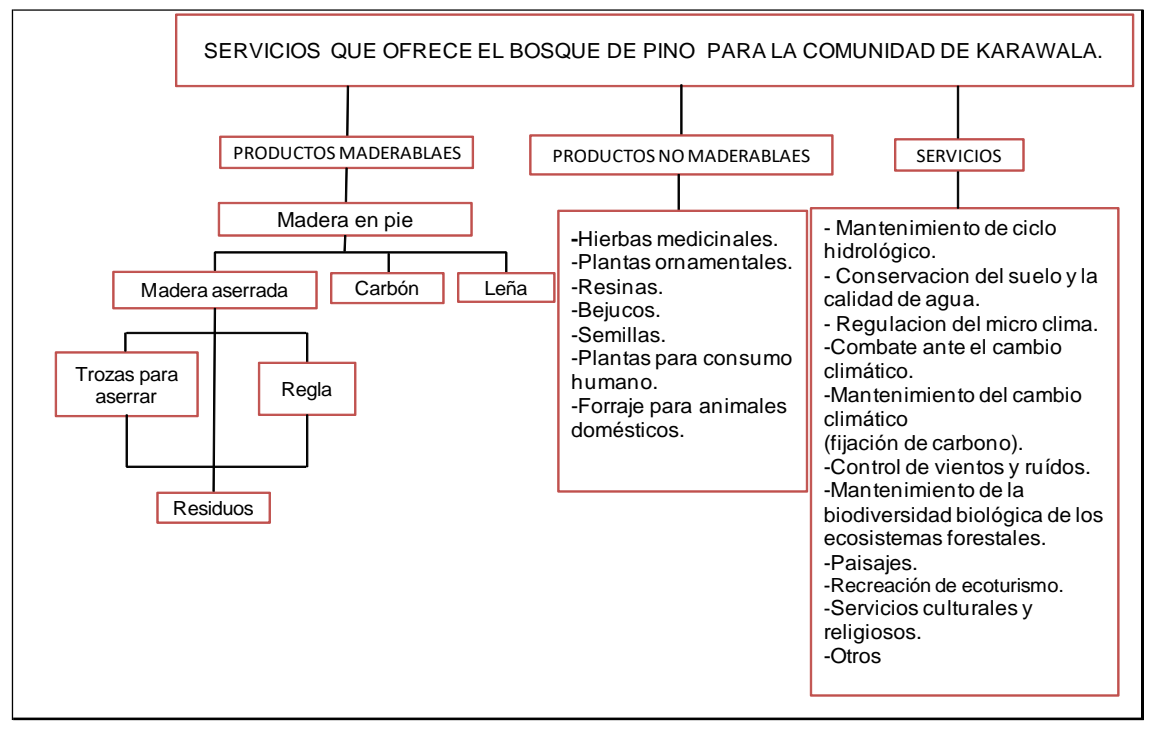

Figura 8. Servicios que ofrece el Bosque de Pino a la comunidad de Karawala.

Las extensiones que cubren estas áreas, y que corresponden específicamente a Karawala, no han sido cuantificadas, pues las mismas son consideradas de propiedad multicomunal por todas las comunidades que conforman el municipio. Sin embargo, el área inmediata más importante para Karawala es el bosque de pino (Pinus caribaea), y se hace necesario realizar acciones, por parte de los líderes comunales, para detener el aprovechamiento ilegal de madera en la zona. El bosque puede ser aprovechado de manera sostenible bajo un manejo adecuado, aunque una buena porción de este ecosistema ya ha sido aprovechada y más bien tiene potencial para ser restaurado. La explotación forestal actual no es sostenible, ya que se realiza con un manejo deficiente y sin la aplicación de prácticas silviculturales 
adecuadas, además existe tala, transporte y venta ilegal de madera dentro y fuera del Municipio.

En base a los estudios realizados en la reserva natural se sugieren las siguientes recomendaciones específicas:

1. Promover la retención de los árboles dispersos/semilleros en los potreros (especialmente de especies riparias) y facilitar la regeneración natural en ellos. La selección y retención de árboles dispersos/semilleros en los potreros es una técnica fácil y barata para aumentar la cobertura arbórea en la Reserva y puede proveer importantes productos, como leña, forraje y madera. La retención de especies forrajeras es de mucha importancia, ya que estos árboles complementan la alimentación del ganado en verano. A la vez, muchos de estos árboles sirven como hábitat o sitio de paso para los animales silvestres.

2. Promover la conservación de los bosques riparios remanentes y sembrar árboles de sombra para aumentar su diversidad y área. Actualmente, los bosques riparios son muy angostos y no protegen adecuadamente el recurso agua ni la biodiversidad. El hecho de que los pobladores que estén interesados en conservar el agua para consumo humano -existe una ley (620) que prohíbe el corte de árboles maderables a la orilla de las quebradas- facilitará la adopción de esta práctica. Se recomienda promover la reforestación con especies propias de los bosques, como Hymenaea courbaril, Pinus caribaea, Pinus tecunumanii, y otras especies maderables como la caoba (Swietenia macrophylla), pino (Pinus caribaea), cedro real (Cedrela odorata), cedro macho (Carapa guianensis).

3. Identificar las áreas agrícolas y de charrales que están en zonas con fuerte pendiente, o cerca de los ríos, y dejar que se regeneren y se recuperen a bosques secundarios. La regeneración natural es la manera más rápida y más barata de recuperar áreas degradadas y aumentar la cobertura arbórea.

4. Enriquecer los charrales o tacotales con especies maderables, para que cuando los pobladores cambien el charral a potrero, permanezcan árboles de uso múltiple que se puedan conservar. El enriquecimiento de charrales es una técnica barata para aumentar la cantidad de árboles que benefician a los productores. En las parcelas de muestreo pudimos localizar subparcelas de monitoreo de las áreas ecológicas, entre ellas, tacotales, charrales y potreros. Durante las mediciones de las cuatro parcelas, y dentro de las subparcelas, pudimos obtener datos representativos que nos muestran la existencia de estas áreas. Dentro de las subparcelas $\mathrm{N}^{\circ} 1$ obtuvimos que un $13.6 \%$ del área es de tacotal; un $16.6 \%$, charrales, y un $69.8 \%$ son potreros. En la subparcela $\mathrm{N}^{\circ} 2$; un $6.6 \%$, tacotales; $18.6 \%$, charrales, y un $74.8 \%$ son potreros. En 
la subparcela $\mathrm{N}^{\circ} 3$, un $20 \%$ son tacotales; $12 \%$, charrales, y un $68 \%$ son potreros. En la subparcela $\mathrm{N}^{\circ} 4$, un $18.6 \%$ son tacotales, $22 \%$ son charrales y $59.8 \%$ son potreros.

5. Promover la implementación de huertos caseros que incluyan especies para el autoconsumo y la venta. La mayoría de los productores disponen de áreas (que podrían ser utilizadas como huertos caseros) y accesibilidad a los mercados. A la vez que contribuyen a la autosuficiencia alimenticia, los huertos aumentarían la cobertura arbórea en el paisaje y servirían como alimento para algunos animales.

6. Fomentar la incorporación de pastos mejorados, con árboles dispersos o pastos de corte o banco de proteína para incrementar la cantidad de biomasa para el ganado y evitar que los productores se vean obligados a cambiar charrales o bosques secundarios a potreros. Actualmente, la cantidad de biomasa de pasto es demasiado baja para sostener el ganado presente y, por lo tanto, el sobrepastoreo y la degradación de las pasturas son comunes. Si los pastos fueran más productivos sería posible que los productores pudieran mantener su ganado en áreas menores y no necesitarían cambiar la cobertura vegetativa (bosques, charrales) a potreros. En la visita a la comunidad de Karawala se realizó un recorrido por diferentes puntos del área para poder focalizar los puntos donde se realizarían los muestreos de campo. En ese recorrido se observó que muchas áreas no cuentan con pasto apto para pastoreo, esto debido al paso de fenómenos naturales como huracanes que devastaron amplios bosques de pinos y áreas de pasto que sí pudieran ser aptos para el pastoreo. Debido a esta problemática, las pocas áreas que aún quedaban fueron sobre pastoradas sin darle un buen manejo o rotación de los mismos.

7. Implementar un aprovechamiento sostenible de la leña y madera extraídas de los parches de bosques, para minimizar el impacto negativo sobre la comunidad vegetativa y animal.

En todas estas actividades es importante tomar en cuenta la situación económica de la comunidad; su visión acerca de cómo quiere manejar los recursos naturales y las actividades que realiza, ya que sus decisiones y actividades definen la composición y estructura del paisaje. Además, estas actividades deben ser implementadas junto con capacitaciones y discusiones con los productores locales, para asegurar que sus intereses y necesidades sean incluidos en cualquier actividad propuesta. Las capacitaciones deben incluir discusiones sobre sistemas agroforestales y silvopastoriles, manejo integrado de bosques y cuencas y la conservación de la biodiversidad. 


\section{CONCLUSIONES}

1. Para la comunidad de Karawala, las especies forestales de mayor extracción selectiva de la reserva natural del Llano son: Pino Caribe (Pinus caribaea), con 70\%; Caoba del Atlántico (Swietenia macrophylla), con 10\%, y Santa María (Calophyllum brasiliense), con 8\%.

2. En el momento de la realización de esta investigación se estimó en $25,500 \mathrm{~m}^{3}$ el volumen de extracción de la especie con más aprovechamiento: Nancitón (Hyeronima alchorneoides). Esta es seguida por la Caoba del Atlántico (Swietenia macrophylla) con $24,000 \mathrm{~m}^{3}$. En contraste, la especie conocida como Santa María (Calophyllum brasiliense) es la que presenta menor extracción, con $16,000 \mathrm{~m}^{3}$ por año.

3. Los servicios y bienes ambientales más destacado en la Reserva son: la captura de carbono por parte del bosque y la protección y mantenimiento de la red hídrica que además es fuente de materia prima para la construcción de viviendas, muebles y medios de transporte para la comunidad y terceros que compran madera extraída directamente del bosque. Estos constituyen un sistema de elementos resilientes para la adaptación y mitigación al cambio climático y permiten una mejor calidad de vida y seguridad alimenticia de la población aledaña. Sin embargo, las mismas actividades humanas están poniendo en riesgo esta situación por la creciente y carente falta de control en torno a la explotación de los recursos de la Reserva.

\section{CONFLICTO DE INTERESES}

Los autores declaran no tener conflicto de intereses.

\section{AGRADECIMIENTO}

Esta investigación fue posible por el apoyo financiero de la Dirección de Investigación y Postgrado (DIP) de la Bluefields Indian \& Caribbean University (BICU). 


\section{REFERENCIAS}

Casasola, F. 2000. Productividad de los sistemas silvopastoriles tradicionales en Moropotente Estelí, Nicaragua. Tesis MSc. CATIE, Turrialba, Costa Rica. 95 p.

Fassbender, H. 1999. "Agroforestería para la producción animal en América Latina". En: Modelos edafológicos de sistemas agroforestales. 2da edición. CATIE. Turrialba. Costa Rica. 490 p.

Harvey, C. 2001. "Síntesis de la Presentación en el Segundo Congreso sobre Agroforestería y Producción de Ganado en América Latina". Segundo Congreso de Agroforestería. San José, Costa Rica. Pág. 80-87.

Hernández I, \& López, C. 2001. Diagnóstico Agro socioeconómico del Municipio de Belén, Departamento de Rivas. NITLAPAN, UCA. Tesis de Licenciatura. Managua, Nicaragua. 82 p.

Herrera, Z., Lanuza B. 1995. Especies para Reforestación en Nicaragua. Servicio Forestal Nacional, Ministerio del Ambiente y Recursos Naturales, MARENA. Tercera Edición Managua, Nicaragua.

Holdridge, L. 1978. Ecología Basada en Zonas de Vida. IICA. San José, Costa Rica. 216 p.

INAFOR/ MARENA. 2009. Definiciones generales para el manejo de un bosque de trópico húmedo.

Instituto Nacional Forestal. 2009. Resultados del Inventario Nacional Forestal: Nicaragua 2007-2008/INAFOR, Managua: INAFOR,.232 p.

Knight L. 2001. Universidad de las Regiones Autónomas de la Costa Caribe Nicaragüense (URACCAN). El Pueblo Ulwa.

Ley No. 690 Art. 5 Definiciones Generales. 01-2007, Reglamento de Áreas Protegidas de Nicaragua, Managua, 2009.

López, J, Claudio, M, y Motto, S. 2006. Estado forestal (Pinus Caribaea), Morelet var. Hondurensis en el pinal, Laguna de Perla RAAS.

Ministerio del medio ambiente y de recursos naturales (MARENA) / CBA. 2003. Sistema Nacional de Áreas Protegidas. 
Ministerio del medio ambiente y de recursos naturales (MARENA) .1999. Dirección General de Biodiversidad y Recursos Naturales, Biodiversidad en Nicaragua un Estudio de País Managua, Nicaragua.

Montagnini, F; et al. 1992. Sistemas agroforestales, principios y aplicaciones en los trópicos. OET, Costa Rica, 622p.

POSAF II/ MARENA. 2005. Manejo y aprovechamiento del bosque Latifoliado Managua, Nicaragua.

Reglamento de áreas protegidas de Nicaragua DECRETO No. 01-2007, Aprobado el 08 de enero del 2007 Publicado en La Gaceta No. 08 del 11 de enero del 2007 el presidente de la república de Nicaragua.

Sampiere, H, Fernández, C, Baptista, L. 2003. Metodología de investigación. 3era edición. Toluca México. pp. 821 


Waini

\section{Ilustraciones}

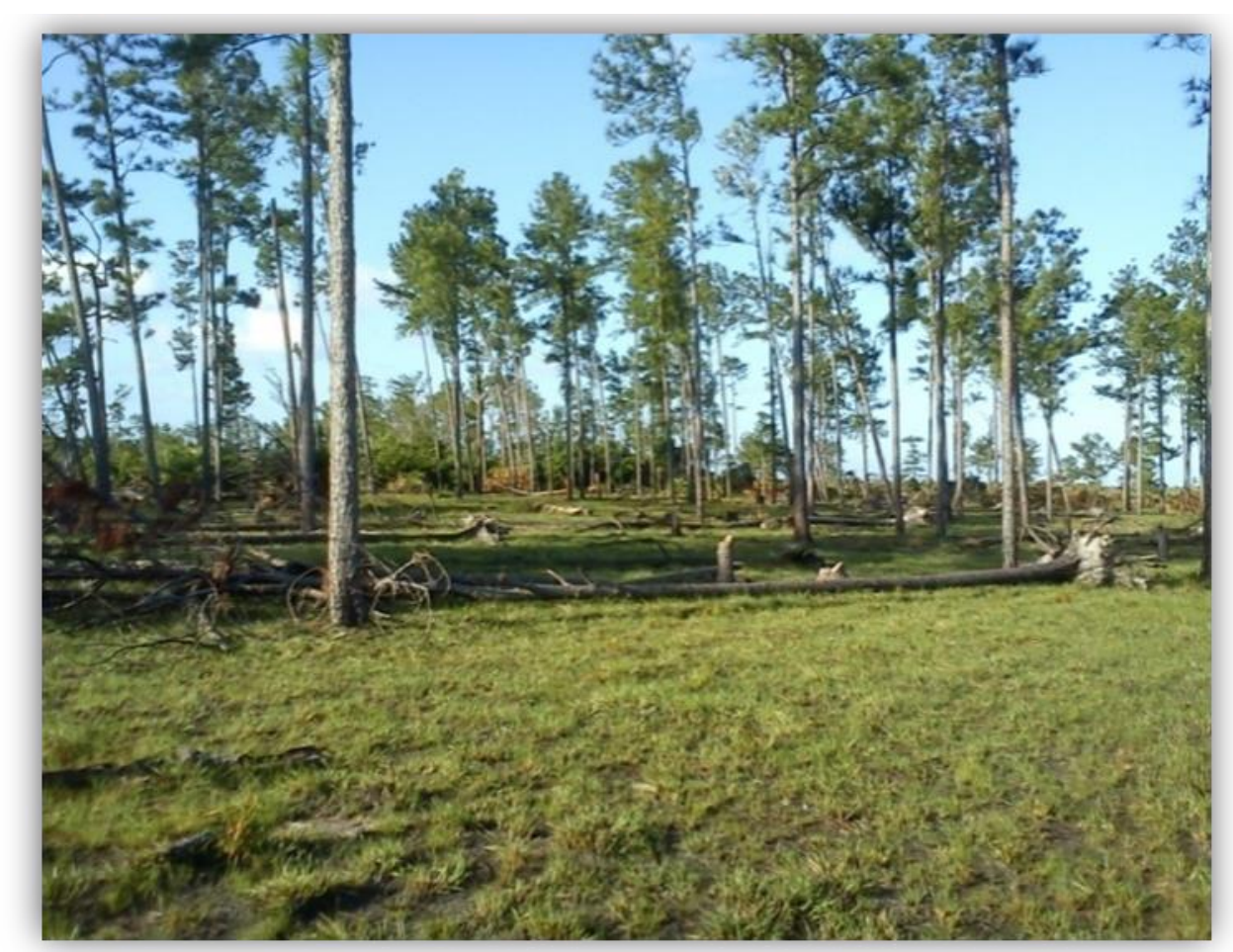

Plantación silvestre de pino caribe (Pinus caribaea) Llanos de Karawala (archivo BICU

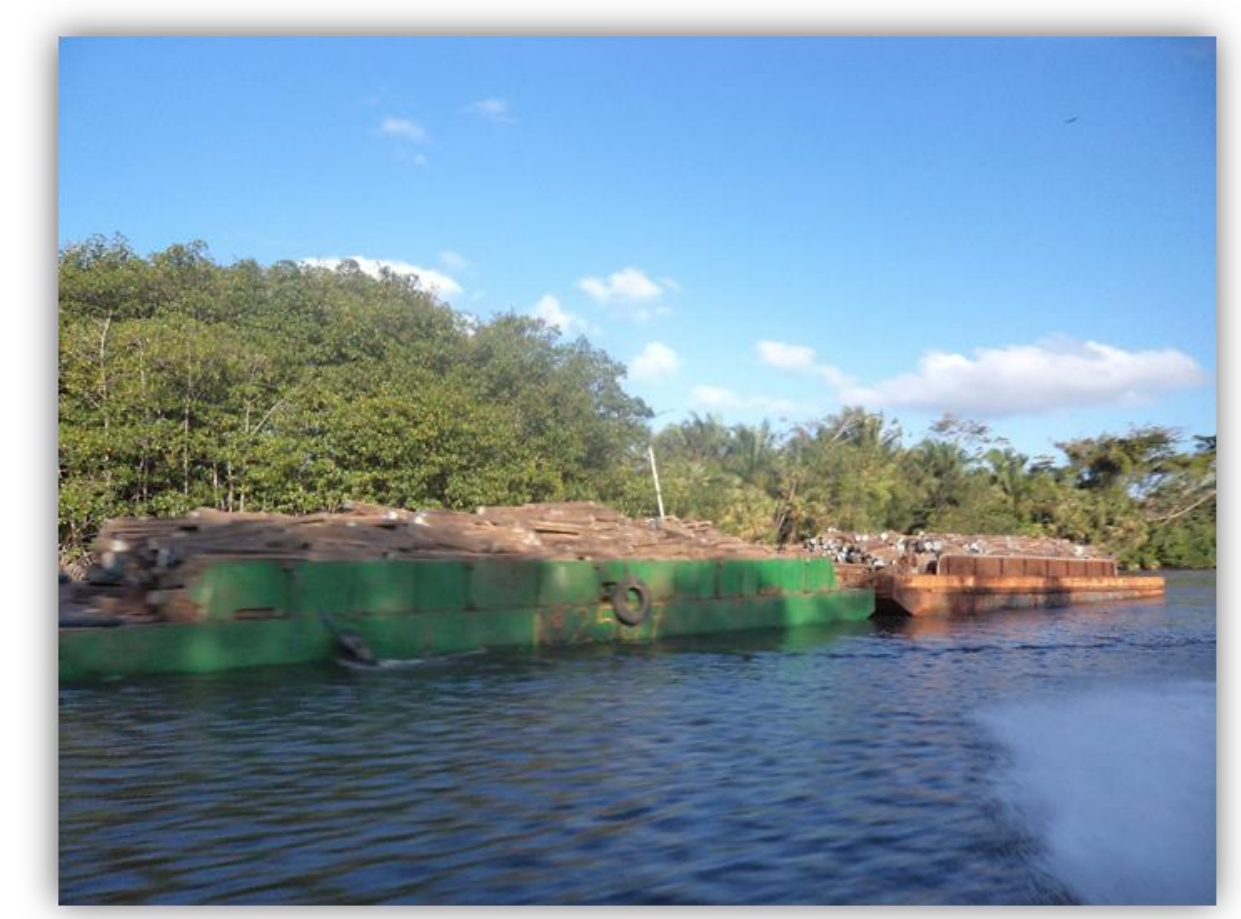

Barcazas privadas con madera extraída de la comunidad de Karawala. (Archivo BICU) 